\title{
Sistema Digital de Bajo Coste para la Monitorización de la Calidad de Energía Eléctrica
}

\author{
João L. Afonso(1), José Batista(2), Manuel J. Sepúlveda ${ }^{(1)}$ y Júlio S. Martins ${ }^{(1)}$ \\ (1) Universidad de Minho, Departamento de Electrónica Industrial, Campus de Azurém, \\ 4800-058 Guimarães-Portugal \\ (2) Instituto Politécnico de Bragança, Escuela Superior de Tecnologia e de Gestão, \\ Campus de Sta. Apolónia, Apartado 134, 5301-857 Bragança-Portugal \\ (e-mail: jla@dei.uminho.pt, jbatista@ipb.pt, mjs@dei.uminho.pt, jmartins@dei.uminho.pt)
}

\begin{abstract}
Resumen
Este artículo describe el desarrollo de un sistema digital de bajo coste utilizado para la monitorización de la calidad de energía eléctrica. El sistema está compuesto por sensores de efecto Hall de tensión y corriente, una tarjeta de adquisición de datos estándar y un ordenador personal vulgar. Las aplicaciones de software han sido desarrolladas en un entorno de programación gráfica (LabVIEW ${ }^{T M}$ ). Varios parámetros, relacionados con la calidad de energía eléctrica y gerencia de la energía, pueden ser analizados por 4 diferentes aplicaciones: "Scope and THD", "Events PQ", "Classical Values" y "p-q Theory". La información capturada pude ser visualizada en tablas y/o gráficos. También es posible generar informes en formato HTML y Word. En formato HTML, los informes pueden ser enviados directamente a una impresora, pueden ser usados en otras aplicaciones de Windows o pueden se consultados por Internet utilizando un navegador Web.
\end{abstract}

Palabras clave: sistema digital, calidad de energía eléctrica, LabVIEW'TM, instrumentación virtual

\section{Low-Cost Digital System for Power Quality Monitoring}

\begin{abstract}
This paper describes the development of a low-cost digital system used for power quality monitoring. The system uses voltage and current Hall sensors, a standard data acquisition board, and has its processing system based on LabVIEW ${ }^{\mathrm{TM}}$, running on a regular Personal Computer. Several parameters related to power quality can be analyzed through 4 different applications: "Scope and THD", "Events PQ", "Classical Values" and "p-q Theory". The acquired information can be visualized in tables and/or in charts. It is also possible to generate reports in HTML format. In this format, the reports can be sent directly to a printer, can be embedded in other software applications, or can be accessed through Internet, using a web browser.
\end{abstract}

Keywords: digital system, electrical energy quality, LabVIEW'TM, virtual instrumentation 


\section{INTRODUCCIÓN}

La calidad de energía eléctrica es hoy en día un tema muy importante que implica los productores de la energía eléctrica, los consumidores y los fabricantes del equipo. Normas mundiales que tratan de la calidad de energía eléctrica (IEEE-519, IEC 61000, EN 50160), obligan que los equipos eléctricos e instalaciones no puedan producir armónicos mayores de los que determinados valores. Sin embargo, los problemas de la calidad de energía eléctrica no son restrictos a los armónicos. El estándar IEEE 1159 clasifica varios fenómenos electromagnéticos en los sistemas de energía, que describen los problemas de la calidad de energía eléctrica: impulsos, oscilaciones, sags, swells, sobre-tensiones, subtensiones, interrupciones, DC offset, armónicos, interarmónicos, microcortes (notches), ruido, flicker, y variaciones de frecuencia (Dugan et al., 1996). Estos problemas se clasifican según la norma IEEE 1159-1995 de la Tabla 1.

La importancia económica de la calidad de energía puede ser cuantificada. En todo el mundo hay muchas compañías donde estos problemas se deben reducir al mínimo para aumentar la productividad (Ross, 2001).

La monitorización de la calidad de energía eléctrica es la mejor manera de detectar y diagnosticar problemas en sistemas de energía eléctrica. Sin embargo, estos equipos son generalmente muy costosos. Los objetivos de este artículo son los de presentar una solución ventajosa y de bajo coste para la monitorización de la calidad de energía eléctrica y análisis de potencia y energía, y también para llamar la atención de la necesidad de mejorar la calidad de la energía eléctrica en los sistemas de potencia modernos.

Tabla 1 - Clasificación de los problemas de la calidad de energía eléctrica.

\begin{tabular}{|c|c|c|c|c|c|}
\hline \multicolumn{4}{|c|}{ Categorias } & $\begin{array}{l}\text { Duración } \\
\text { Típica }\end{array}$ & $\begin{array}{l}\text { Amplitud } \\
\text { Típica }\end{array}$ \\
\hline \multirow{6}{*}{\multicolumn{2}{|c|}{ Transitório }} & \multirow{3}{*}{ Impulsos } & Nanosegundos & $<50 \eta \mathrm{s}$ & \\
\hline & & & Microsegundos & $50 \eta \mathrm{s}$ a $1 \mathrm{~ms}$ & \\
\hline & & & Milisegundos & $>1 \mathrm{~ms}$ & \\
\hline & & \multirow{3}{*}{ Oscilaciónes } & Alta Frecuencia & $5 \mu \mathrm{s}$ & 0 a 4 pu \\
\hline & & & Frecuencia Intermedia & $20 \mu \mathrm{s}$ & 0 a 8 pu \\
\hline & & & Baja Frecuencia & 0,3 a $50 \mathrm{~ms}$ & 0 a 4 pu \\
\hline \multirow{8}{*}{$\begin{array}{c}\text { Variación de } \\
\text { Pequeña } \\
\text { Duración }\end{array}$} & \multirow{2}{*}{ Instantâneas } & & Sag & 0,5 a 30 ciclos & 0,1 a 0,9 pu \\
\hline & & & Swell & 0,5 a 30 ciclos & 1,1 a $1,8 \mathrm{pu}$ \\
\hline & \multirow{3}{*}{ Momentâneas } & & nterrupción & 0,5 ciclos a $3 \mathrm{~s}$ & $<0,1 \mathrm{pu}$ \\
\hline & & & Sag & 30 ciclos a $3 \mathrm{~s}$ & 0,1 a 0,9 pu \\
\hline & & & Swell & 30 ciclos a $3 \mathrm{~s}$ & 1,1 a $1,4 \mathrm{pu}$ \\
\hline & \multirow{3}{*}{ Temporárias } & & nterrupción & $3 \mathrm{~s}$ a $1 \mathrm{~min}$. & $<0,1 \mathrm{pu}$ \\
\hline & & & Sag & $3 \mathrm{~s} \mathrm{a} 1 \mathrm{~min}$. & 0,1 a $0,9 \mathrm{pu}$ \\
\hline & & & Swell & $3 \mathrm{~s}$ a $1 \mathrm{~min}$. & 1,1 a 1,2 pu \\
\hline \multirow{3}{*}{\multicolumn{2}{|c|}{$\begin{array}{c}\text { Variación de } \\
\text { Luenga Duración }\end{array}$}} & Interru & oción Persistente & $>1 \mathrm{~min}$. & $0,0 \mathrm{pu}$ \\
\hline & & & Subtensión & $>1 \mathrm{~min}$. & 0,8 a 0,9 pu \\
\hline & & & obretensión & $>1 \mathrm{~min}$ & 1,1 a 1,2 pu \\
\hline \multicolumn{4}{|c|}{ Desbalanceo de Tension } & Régimen Permanente & 0,5 a $2 \%$ \\
\hline \multirow{5}{*}{\multicolumn{2}{|c|}{$\begin{array}{l}\text { Distorsión de la } \\
\text { Forma de Onda }\end{array}$}} & & Offset DC & Régimen Permanente & 0,0 a $0,1 \%$ \\
\hline & & & Armónicos & Régimen Permanente & 0 a $20 \%$ \\
\hline & & & terarmónicos & Régimen Permanente & 0 a $2 \%$ \\
\hline & & & Notching & Régimen Permanente & \\
\hline & & & Ruído & Régimen Permanente & 0 a $1 \%$ \\
\hline \multicolumn{4}{|c|}{ Fluctuación de Tension } & Intermitente & 0,1 a $7 \%$ \\
\hline \multicolumn{4}{|c|}{ Variación de Frecuencia } & $<10 \mathrm{~s}$ & \\
\hline
\end{tabular}




\section{DESCRIPCIÓN DEL HARDWARE}

El sistema de medida utiliza sensores del efecto Hall - 4 sensores de tensión y 4 sensores de corriente para medir las tensiones de fase-neutro $\left(v_{a n}, V_{b n}, v_{c n}\right)$ y neutro-tierra $\left(v_{n g}\right)$ y corrientes de las fases $\left(i_{a}, i_{b}, i_{c}\right)$, y la corriente del conductor neutro $\left(i_{n}\right)$. La salida de los sensores son señales de pequeña amplitud en tensión. Además, los sensores proporcionan aislamiento galvánico del sistema eléctrico, lo que permite una operación segura con el sistema de monitorización desarrollado y presentan una muy buena linealidad.

El equipo de medida está basado en un ordenador personal estándar (con sistema operativo Windows XP) con una tarjeta de adquisición de datos para bus PCl (MIO-PCl-6024E de la National Instruments). Las características más significativas son: 16 canales analógicos (8 canales en modo diferencial), 12 bits de resolución, 200 kmuestras/s, 8 líneas digitales de I/O, FIFO de 512 muestras, y posibilidad de adquisición de datos por DMA (Direct Memory Access).

\section{SISTEMA DE MONITORIZACIÓN DESARROLLADO}

En la Fig. 1, se muestra un diagrama con la arquitectura del sistema desarrollado (Batista et al., 2003). El software del sistema de monitorización ha sido desarrollado entorno de programación gráfica LabVIEW (de la National Instruments). El sistema adquiere datos continuamente, y almacena en ficheros las incidencias que resultan de la detección de anomalías en el sistema de energía monitorizado (varios parámetros relacionados con la calidad de la energía son analizados). La información conseguida se puede visualizar en tablas y/o en gráficos. También es posible generar informes en formato HTML. Después de la adquisición, procesado y análisis, datos y alarmes se pueden enviar a través de Internet o utilizando tecnología Wireless (Travis, 2000).

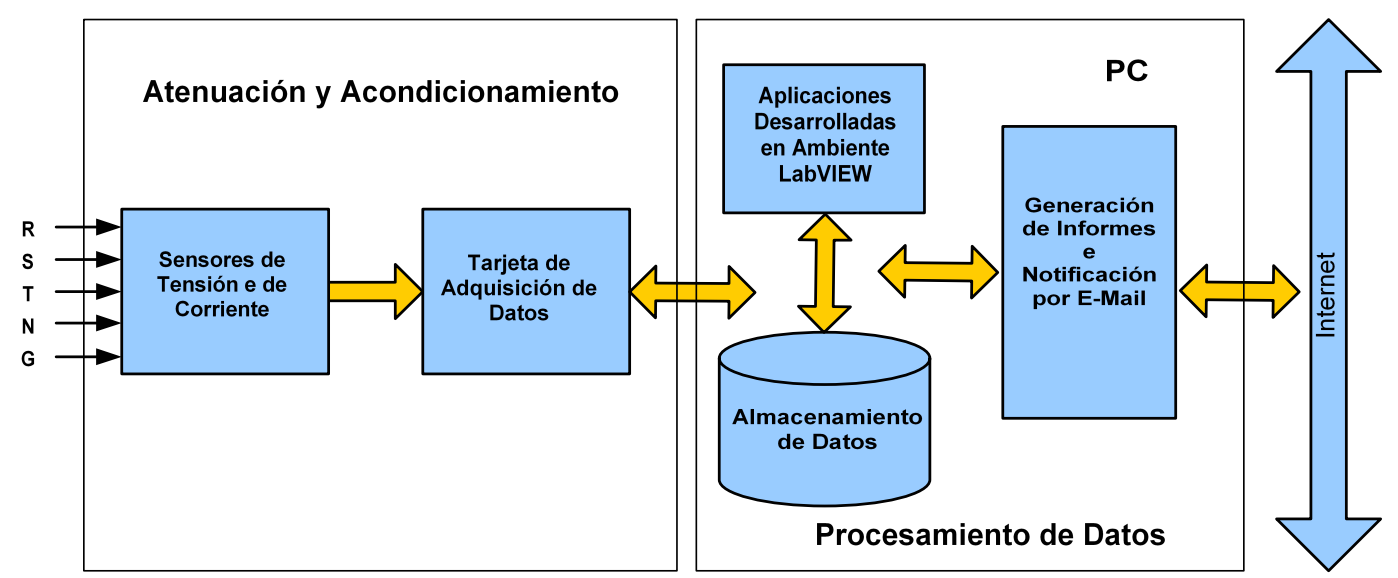

Fig. 1: Arquitectura del sistema de monitorización.

En la Fig. 2, se muestra lo sistema de monitorización funcionando en una instalación eléctrica de una fabrica.

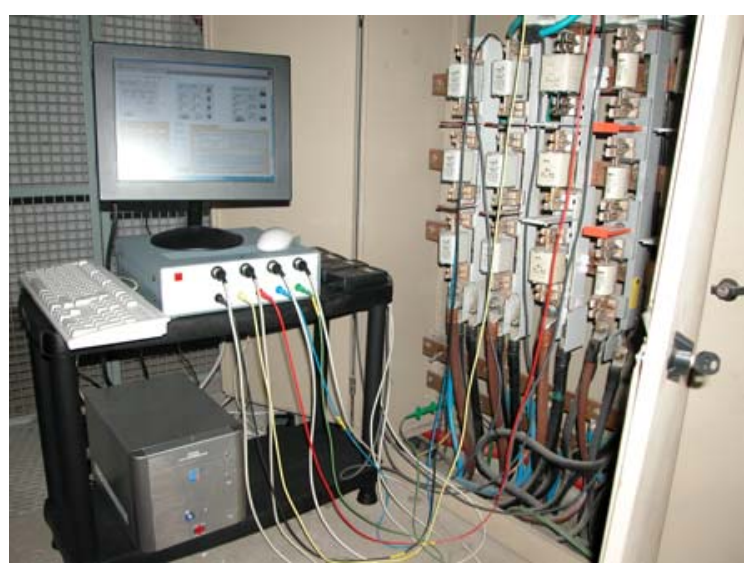

Fig. 2: Sistema de monitorización instalado. 


\section{APLICACIONES DESARROLLADAS EN LABVIEW}

El nombre de un programa desarrollado en LabVIEW es un VI (Virtual Instrument), porque su aspecto y el funcionamiento se asemejan a los instrumentos verdaderos, como osciloscopios y multímetros. Los VIs utilizan funciones que manipulan la información que viene del utilizador, hacen cálculos, presentan los datos empleando la pantalla del ordenador o los pueden transferir para otros ordenadores. Después de la definición de las características generales y de las especificaciones del sistema, fue seguida una metodología top-down y modular para el desarrollo del software. Fueron elaboradas 4 aplicaciones: "Scope and THD", "Events PQ", "Classical Values" y la aplicación "Theory p-q".

Las aplicaciones desarrolladas consisten en diferentes módulos de software. Dentro de cada módulo hay un primer nivel jerárquico que contiene el VI principal. Descendiendo en la jerarquía, dentro de cada nivel, pueden ser alcanzadas otras operaciones, que pueden ser manipuladas por el usuario a través de menús y menús de nivel secundario.

El sistema desarrollado permite la monitorización, medida y análisis de diversos parámetros del sistema de energía observado, seleccionando la aplicación deseada. Abajo, se describe las aplicaciones desarrolladas.

\section{Aplicación "Scope and THD"}

Esta aplicación es un ejemplo típico de la instrumentación virtual, porque imita un Scope digital con 8 canales aislados con las funciones básicas: base del tiempo, amplificador vertical, factores multiplicativos, disposición del trigger (slope, level y source) y función de readouts (frecuencia de las señales, verdadero valor eficaz ( $T_{\text {RMS }}$ ), valores $D C$, valores pico a pico y otros. Es importante destacar que este instrumento calcula el verdadero valor eficaz, puesto que muchos instrumentos de hecho calculan un "falso" valor RMS, basado en cálculo del valor medio de la forma de onda rectificada (supuesta para ser sinusoidal), y en el valor del factor de cresta (la razón entre el RMS y el valor medio).

Con esta aplicación es posible visualizar hasta 8 señales simultáneamente, con 25 kmuestras/s por canal. Es también posible calcular los armónicos y el THD (Total Harmonic Distortion) de cualquier señal deseada.

El sistema puede también generar informes en formato HTML y registrar datos en archivos que se pueden manipular por otras herramientas de software estándar que funcionen en Windows, como Excel o Matlab.

La Fig. 3 presenta la interface de usuario del aplicación Scope and THD como aparece en la pantalla del ordenador.

Nuevas funcionalidades se pueden agregar fácilmente a esto sistema, aprovechándose la programación gráfica y el uso del potencial de los subVIs en LabVIEW.

\section{Aplicación “Events PQ” (Power Quality)}

Esta aplicación ejecuta, en modo continuo, la adquisición de las 3 señales de la tensión fase-neutro (van, vbn, vcn), de la señal de tensión neutro-tierra (vng), de 4 señales de corriente (corriente de las fases ia, ib, ic) y corriente del neutro (in). La aplicación ejecuta, en simultáneo, todo el proceso necesario para 4 funciones diferentes: elaborar Strip Charts, detectar perturbaciones (Sags and Swells), detectar anomalías Wave Shape (medida de la forma de onda) y medir la energía activa e reactiva. El aspecto crítico en la implementación y ejecución de esta aplicación, consiste en el hecho de que debe funcionar continuamente sin perder muestras. El sistema desarrollado ha sido probado en diversas situaciones y para periodos largos de tiempo, confirmándose una correcta operación.

La función de Strip Chart permite el cálculo, el registro y la monitorización de los valores RMS a lo largo del tiempo, generalmente para intervalos largos del tiempo. Puede registrar los valores RMS de las 4 señales de tensión y 4 señales de corriente. La corriente del conductor tierra no se mide, 


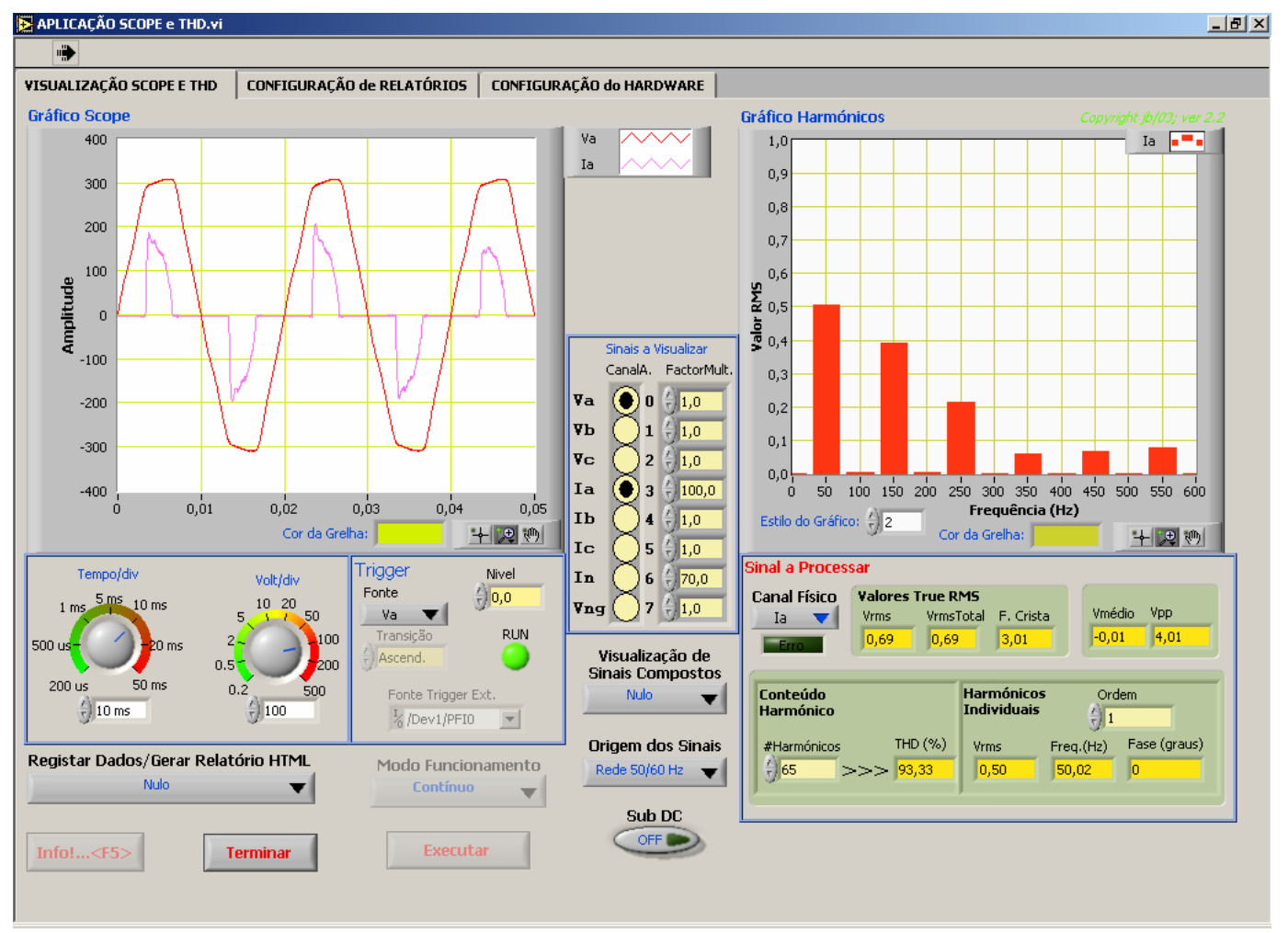

Fig. 3: Interface de usuario del aplicación "Scope and THD".

por que puede ser calculada de las corrientes de las fases y del neutro. El usuario puede fijar el tiempo de Strip Chart a partir de 1 minuto a 31 días. Las señales se pueden visualizar online y la aplicación puede ser interrumpida en cualquier momento (en cualquier caso los datos se almacenan siempre en disco duro del ordenador). El programa realiza los cálculos del RMS en cada ciclo y el intervalo del tiempo de los datos registrados se puede ajustar por el usuario, evitando almacenaje de datos en exceso. La Fig. 4 presenta la información producida para elaborar Strip Charts.

La función de Wave Shape detecta distorsiones en las formas de onda del voltaje de las 3 fases y registra estas anomalías en fichero. En adquisición continua, los valores de las muestras del ciclo $n$ (señales de tensión) se comparan con los valores de las muestras del ciclo n-1. Si el resultado de la comparación excede un límite previamente establecido (dado por uno valor de tolerancia especificado), se considera que ha ocurrido una anomalía, y la incidencia (event) se coloca en fichero. Hay siempre dos ciclos de las señales del voltaje que son procesados. Cuando se detecta un disturbio, las muestras de estos dos ciclos se registran las muestras de dos ciclos antes de ellos y de dos ciclos después de ellos. Por lo tanto, cuando se detecta una anomalía, son registrados 6 ciclos. Esto que se registra se hace para los 3 voltajes de las fases, incluso si la anomalía se detecta solamente en una de las fases. La Fig. 5 presenta un ejemplo de distorsión (Wave Shape) registrada por la aplicación Events $P Q$.

La función Sags and Swells detecta y registra en fichero esas ocurrencias, de corta y larga duración. Simultáneamente, el programa compara los valores medidos con los valores especificados de los voltajes fase-neutro (van, vbn, vcn) y voltaje neutro-tierra (vng). Las tolerancias de funcionamiento son introducidas por el usuario, que puede aplicar diversos estándares en lo referente al comportamiento aceptado de voltajes medidos.

\section{Aplicación "Classical Values"}

Esta aplicación calcula los valores RMS de la amplitud y los ángulos de la fase de cada tensión y de cada corriente. También calcula la amplitud y el ángulo de la impedancia (por fase). Además de los valores numéricos, la misma información se puede analizar visualmente (véase la Fig. 6). También se calculan el desequilibrio del voltaje y corriente, así como los factores de potencia total y 


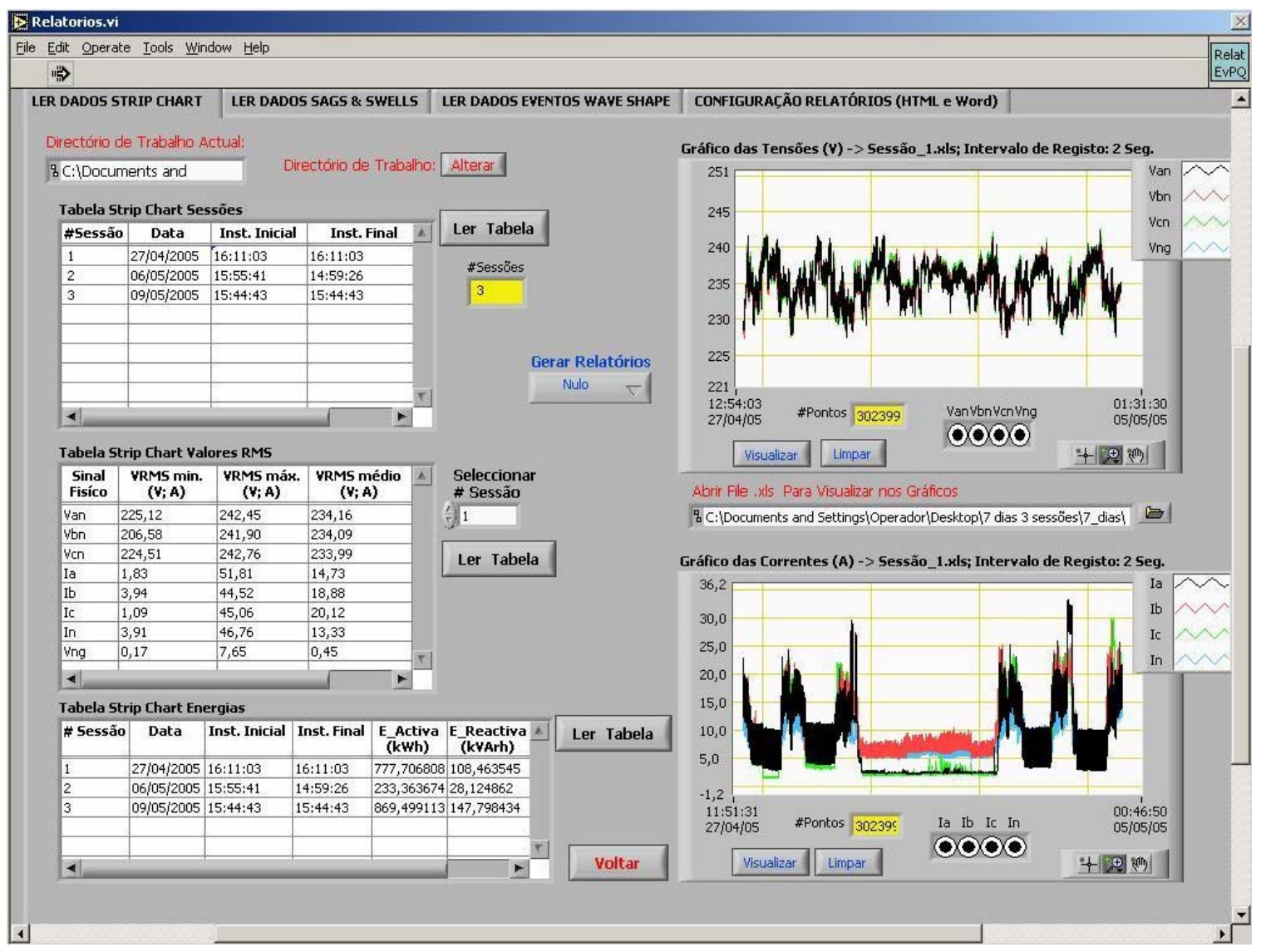

Fig. 4: Strip Chart generado por la aplicación "Events PQ".

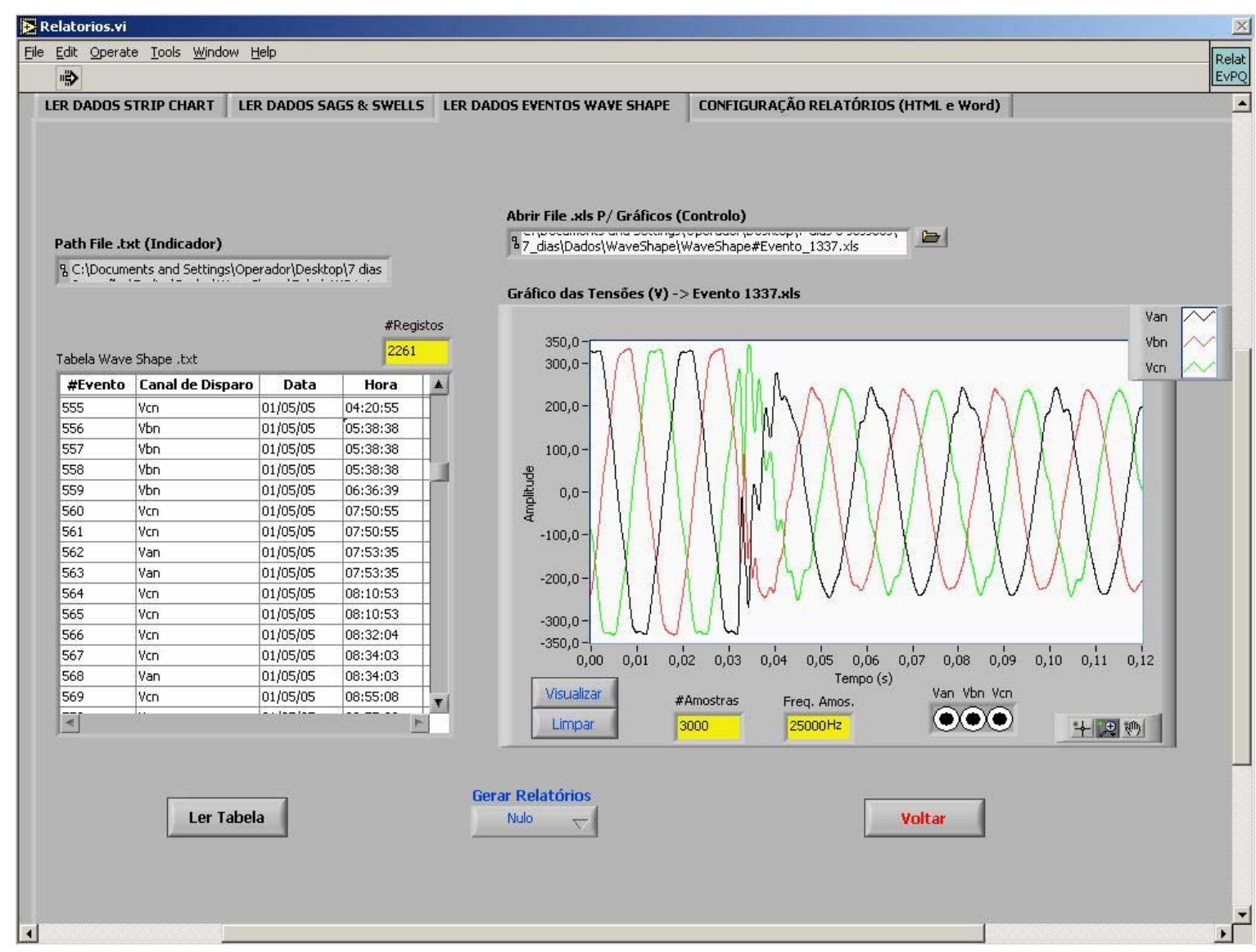

Fig. 5: Distorsión (Wave Shape) registrada por la aplicación "Events PQ". 
dislocación. Además, también se registran los valores de kWh (energía activa) y kVArh ("energía" reactiva). Esta aplicación funciona en dos modos: "Adquisición" y "Simulación". En el modo "Simulación" se hacen todos los cálculos basados en las señales generadas en LabVIEW. Los parámetros de estas señales (amplitud, ángulo y frecuencia) son configurados por el usuario, y es posible sintetizar una señal con una frecuencia fundamental y sus armónicos. En modo "Adquisición" los cálculos se hacen con las señales verdaderas, adquiridas por los sensores de tensión y corriente.

\section{Aplicación "p-q Theory"}

Esta aplicación calcula y muestra, numérica y gráficamente, algunos de los valores obtenidos por la Teoría p-q (Afonso et al., 2000). Esta teoría, también conocida como "Teoría de la potencia instantánea" fue propuesta por Akagi et al. (1983) para controlar los filtros activos. Se basa en los valores instantáneos de tensión y corriente en sistemas de potencia trifásicos con o sin conductor neutro, y es válida para la operación de estado estacionario o transitorio, así como para formas de onda genéricas de tensión y corriente. La Teoría p-q consiste básicamente en la transformación algebraica (transformación de Clarke) de la tensión y corriente del sistema trifásico de coordenadas a-b-c en coordenadas $\alpha-\beta-0$, seguido por los cálculos de los componentes instantáneos de potencia de la Teoría p-q. El conocimiento de estos componentes es muy útil para analizar sistemas de energía, además de permitir una comprensión fácil de varios problemas de la calidad de la energía (armónicos, energía reactiva, desequilibrios de la carga, etc) en sistemas trifásicos (Costa et al., 2005).

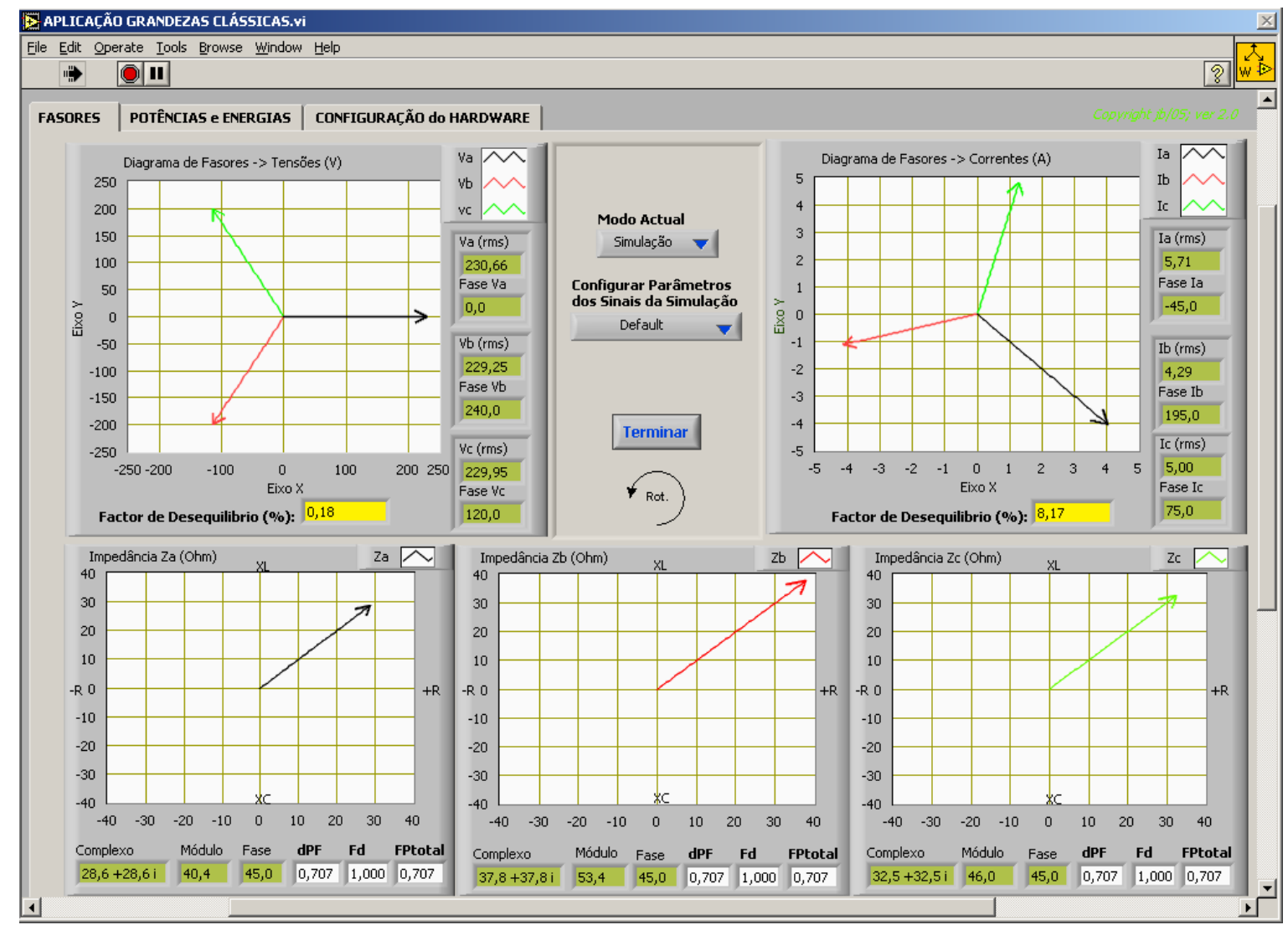

Fig. 6: Interface del usuario con la aplicación "Classical Values".

\section{COMPARACIÓN CON LOS SISTEMAS COMERCIALES}

Después de investigar los equipamientos destinados al registro y evaluación de la calidad de la energía eléctrica comercialmente disponibles, se puede concluir que, los actuales productos desarrollados por los principales fabricantes, presentan básicamente los aspectos siguientes:

- Los productos comerciales de línea superior son generalmente muy costosos. 
- Las paquetes de software desarrolladas específicamente por cada fabricante, tecnología del Internet a veces que explora, enriquecen la gerencia de la energía y la supervisión de la calidad de la energía, permitiendo el desarrollo de aplicaciones distribuidas.

- Las características y las funciones de los equipos son generalmente muy buenas en la línea superior de productos.

Algunos fabricantes presentan un equipo bajo con varios módulos opcionales. El software y los accesorios deben también ser comprados. Por ejemplo, un módulo bajo capaz de realizar el análisis de los armónicos, cálculos dos valores RMS, y la detección de los sags e swells puede costar unos $600 €$. Un módulo opcional para la detección de transitorios puede extenderse del $2000 €$ al $5000 €$. Un módulo para la gerencia de la energía eléctrica puede costar unos $2500 €$.

El software del sistema (imprescindible) puede costar $1000 €$ y el software para permitir la generación de informes, otros $1000 €$. Por lo tanto, deducimos que un sistema completo, con buenas características, puede costar de $15000 €$ a $20000 €$, o más.

Es importante destacar que los costes los equipamientos comerciales dependen de sus características y funciones, y también en los fabricantes. Hay también productos que se extienden de precios tan bajos como el $700 €$ al $3000 €$, pero pueden ser muy limitados, y es generalmente necesario utilizar un ordenador para descargar los datos registrados de estos equipos, para realizar la análisis de los datos y generación de informe.

El sistema de monitorización propuesto se puede considerar una solución barata, puesto que, además del ordenador, utiliza 4 sensores de tensión y 4 sensores de corriente, de efecto Hall, con un coste total alrededor del $280 €$, y una tarjeta de adquisición de datos y sus accesorios, que tienen un coste total casi del $400 €$. El precio del a tarjeta de circuito impreso y de algunos componentes electrónicos discretos necesarios en el módulo de hardware es insignificante en lo referente a los costes restantes. Los precios de los ordenadores personales disponibles en el mercado están disminuyendo siempre, y puesto que no es necesario utilizar un modelo superior para el sistema de supervisión, se puede utilizar un ordenador prescindible, con costes adicionales mínimos. Este sistema presenta también características de elevado potencial:

- Gran flexibilidad y funciones adicionales con respecto a los productos comerciales. Adquisición y visualización de hasta 8 canales independientes. La velocidad de adquisición es limitada solo por la tarjeta de adquisición, que permite 25.000 muestras/s por canal si todos los canales se utilizan simultáneamente, hasta 200.000 muestras/s si se utiliza solo uno. Las entradas/salidas digitales pueden ser utilizadas para control de procesos, activación de alarmas, comunicación con periféricos, etc.

- Los datos se almacenan en el disco duro del ordenador. La solución propuesta sobrepasa la mayoría de productos comerciales con respecto a la capacidad para almacenar datos.

- La solución propuesta presenta un ambiente de trabajo amistoso con el usuario. Puesto que trabaja en un ordenador con un sistema operativo Windows, el usuario puede manejar el sistema con la ayuda del teclado, del ratón y de un monitor coloreado grande. Los interfaces son ventanas con las ventajas de los procedimientos del point-and-click.

\section{CONCLUSIONES}

Hay varios tipos de equipos en el mercado que se pueden utilizar para monitorizar la calidad de la energía eléctrica, o para realizar estudios del consumo de energía. Sin embargo, son generalmente muy costosos.

Este artículo demuestra que es posible desarrollar un equipo muy útil para la gerencia y monitorización de la calidad de la energía eléctrica, conveniente para instalaciones industriales o comerciales, de coste reducido. 
También se presenta la utilización de la Teoría p-q en el análisis de los sistemas de energía trifásicos. Este análisis ha sido incluido en el equipo desarrollado, porque puede ser muy útil para diagnosticar los problemas relacionados con la calidad en los sistemas eléctricos.

\section{AGRADECIMIENTOS}

Los autores agradecen a la FCT (Fundação para a Ciência e a Tecnologia), por financiamiento con fondos FEDER y OE del Proyecto POCTI/ESE/41170/2001.

\section{REFERENCIAS}

Afonso, J., C. Couto y J. Martins; Active Filters with Control Based on the $p$ - $q$ Theory, IEEE Industrial Electronics Society Newsletter, vol.47, n³, pp. 5-10, Sept. (2000)

Akagi, H, Y. Kanazawa y A. Nabae; Generalized Theory of the Instantaneous Reactive Power in Three-Phase Circuits, IPEC'83 - Int. Power Electronics Conf., Tokyo, Japan, pp. 1375-1386 (1983)

Batista, J., J.S. Martins y J.L. Afonso; Low-Cost Power Quality Monitor Based on a PC, ISIE'2003 IEEE International Symposium on Industrial Electronics, Rio de Janeiro, Brasil, 9-11 Junho (2003)

Costa, J.C.C., R.G. Pregitzer, T.N. Sousa y J.L. Afonso; A Case of Power Quality Assessment Using a Developed Power Quality Monitor, CEE'05, Coimbra, Portugal, 10-12 Out. (2005)

Dugan, R.C., M.F. MacGranaghan y H. Wayne; Electrical Power Systems Quality, MacGraw-Hill (1996)

Ross, R., T. Assis y L. Brasil; Investigação da Qualidade de Energia Eléctrica em Clientes Industrias da RGE, IV SBQEE, Porto Alegre, Brasil (2001)

Travis, J.; Internet Aplications in LabVIEW, Prentice Hall (2000) 